\title{
Escalating Dose Methamphetamine Pretreatment Alters the Behavioral and Neurochemical Profiles Associated with Exposure to a High-Dose Methamphetamine Binge
}

\author{
David S Segal*,', Ronald Kuczenski', Meghan L O'Neil², William P Melega² and Arthur K Cho \\ 'Department of Psychiatry, University of California, San Diego, School of Medicine, La Jolla, CA, USA; ${ }^{2}$ Department of Molecular and Medical \\ Pharmacology, University of California, Los Angeles, School of Medicine, Los Angeles, California, USA
}

\begin{abstract}
The neurotoxic effects of methamphetamine (METH) have been characterized primarily from the study of high-dose binge regimens in rodents. However, this drug administration paradigm does not include a potentially important feature of stimulant abuse in humans, that is, the gradual escalation of stimulant doses that frequently occurs prior to high-dose exposure. We have argued that pretreatment with escalating doses (EDs) might significantly alter the neurotoxic profile produced by a single high-dose binge. In the present study, we tested this hypothesis by pretreating rats with saline or gradually increasing doses of METH $(0.1-4.0 \mathrm{mg} / \mathrm{kg}$ over 14 days), prior to an acute METH binge $(4 \times 6 \mathrm{mg} / \mathrm{kg}$ at $2 \mathrm{~h}$ intervals). These animals, whose behavior was continuously monitored throughout drug treatment, were then killed 3 days later for determination of caudate-putamen dopamine (DA) content, levels of $\left[{ }^{3} \mathrm{H}\right] \mathrm{WIN} 35,428$ binding to the DA transporter, and levels of $\left[{ }^{3} \mathrm{H}\right]$ dihydrotetrabenazine $\left(\left[{ }^{3} \mathrm{H}\right] \mathrm{DTBZ}\right)$ binding to the vesicular monoamine transporter. ED pretreatment markedly attenuated the stereotypy response, as well as the hyperthermia and indices of sympathetic activation associated

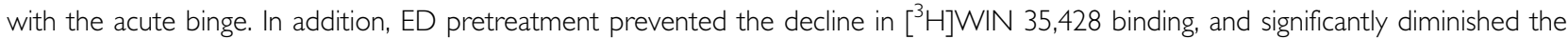
decrease in DA levels, but did not affect the decrease in $\left.{ }^{3} \mathrm{H}\right] \mathrm{DTBZ}$ binding associated with the acute binge. We suggest that further study of the effects produced by a regimen which includes a gradual escalation of doses prior to high-dose METH binge exposure could more accurately identify the neurochemical and behavioral changes relevant to those that occur as a consequence of high-dose METH abuse in humans.
\end{abstract}

Neuropsychopharmacology (2003) 28, 1730-1740, advance online publication, 09 July 2003; doi: I0.1038/sj.npp. 1300247

Keywords: dopamine; methamphetamine; neurotoxicity; dopamine transporter; vesicular monoamine transporter

\section{INTRODUCTION}

Amphetamine-induced psychosis is most commonly associated with daily, multiple high-dose drug administrations, and therefore, an understanding of the neurobiological effects produced by this stimulant exposure pattern might provide an insight into the pathophysiology of naturally occurring psychosis (Angrist, 1994; Segal and Kuczenski, 1997a). In this regard, accumulating evidence suggests that neurotoxic effects may result from this form of stimulant abuse and therefore, such changes could contribute to the frequently observed augmented sensitivity to the psychosisinducing effects of these drugs. Recently, the results of a number of studies in humans, including both post-mortem

\footnotetext{
*Correspondence: Dr DS Segal, Department of Psychiatry (0603), UCSD School of Medicine, La Jolla, CA 92093, USA, Tel: + I 858534 4044, Fax: + I 858534 7653, E-mail: dsegal@ucsd.edu

Received 19 April 2003; revised 12 May 2003; accepted 21 May 2003 Online publication: 22 May 2003 at http://www.acnp.org/citations/ Npp5223। I5/default.pdf
}

and neuroimaging indices of various regional brain neurochemical processes, have indicated that pronounced effects may persist long after cessation of drug administration, particularly in high-dose methamphetamine (METH) abusers (Villemagne et al, 1998; McCann et al, 1998; Volkow et al, 1999, 2001a; Sekine et al, 2001, 2002; Chang et al, 2002). As dopamine (DA) systems have been implicated in many effects of stimulants, including psychosis, considerable interest has been focused on the impact of METH abuse on various DA terminal markers. In fact, some evidence showing long-term declines in the levels of the dopamine transporter (DAT) have been interpreted as reflecting permanent dysfunction in DA transmission in selective DA pathways (McCann et al, 1998; Volkow et al, 1999; Sekine et al, 2001).

However, multiple interpretations exist regarding the nature of these neurochemical changes, that is, whether they reflect compensatory alterations or neuronal damage, as well as the degree to which they exhibit, at least, functional recovery over time (Wilson et al, 1996; McCann et al, 1998; Harvey et al, 2000; Guilarte, 2001; Davidson et al, 2001; 
Volkow et al, 2001b). In part, variations in persistence of changes observed in METH abusers may stem from the presence of numerous potentially confounding factors, including concurrent abuse of other drugs such as alcohol and/or marijuana, as well as the reliance on self-reports for characterization of drug dose and usage pattern (Angrist, 1994; Gawin and Khalsa, 1996; Huber et al, 1997; Simon et al, 2000). Therefore, despite continuing technological advances in detecting drug-induced changes in vivo, the relative uncertainty regarding details known to influence stimulant effects may significantly compromise the accurate interpretation of results and likely contributes to the variability in the findings reported in METH abusers.

To overcome many of these difficulties, various animal models have been proposed using specific drug exposure protocols. In addition to the ability to precisely control dosing regimens, studies in animals allow for a significantly greater range and accuracy in the neurochemical characterization of stimulant effects. Particularly with respect to rodent models of METH abuse, an acute 'binge' regimen has been most frequently used to study the consequences of high-dose METH exposure. The high doses used (most commonly in the range of $4-10 \mathrm{mg} / \mathrm{kg}$ ) are presumed to be comparable to those achieved by many long-term METH abusers (see, eg, Sonsalla and Heikkila, 1988; Hogan et al, 2000; Davidson et al, 2001). Multiple injections are administered within a day, typically at 2 -h intervals, to simulate the prolonged elevation of drug associated with binge-pattern administration in humans. Although several variations in this procedure have been studied, most researchers have reported a 'neurotoxic' profile which, especially at the higher doses, includes regionally specific damage to DA terminals as well as profound, although less persistent, changes in other neurotransmitter systems (Seiden and Ricaurte, 1987; Eisch et al, 1992; Axt et al, 1994; Bowyer and Holson, 1995; Pu and Vorhees, 1995; Cass, 1997; Fleckenstein et al, 2000; O'Callaghan and Miller, 2000). The mechanisms underlying these changes have received considerable attention, with many studies suggesting important roles for temperature and accumulation of reactive oxidative species, as well as other factors, in the cascade of changes that appear to result from this high-dose 'binge' protocol (Farfel and Seiden, 1994; Seiden and Sabol, 1995; Stephans and Yamamoto, 1996; Wrona et al, 1997; Xie et al, 2000; Davidson et al, 2001; Yuan et al, 2001; Larsen et al, 2002).

Although these studies have stimulated considerable interest in the neurotoxic effects that can result from high-dose METH-like drugs, we have argued that the drug administration paradigm typically used in this research does not include a relevant feature of stimulant abuse in humans (Segal and Kuczenski, 1997a,b,c; Segal and Kuczenski, 1999a,b). That is, most high-dose METH abusers initially use lower doses, administered at relatively long intervals, before progressively increasing the dose and reducing the interval between successive administrations, eventually engaging in multiple daily administrations (Kramer, 1972; Angrist, 1994; Gawin and Khalsa, 1996; Huber et al, 1997; Simon et al, 2002). Importantly, the increased levels of stimulant exposure are achieved in the absence of many of the toxic effects that are typically associated with high acute stimulant doses, for example, daily intake of up to several grams in humans occurs without lethality (Angrist, 1994; Lit et al, 1996; Volkow et al, 1999; Davidson et al, 2001; Simon et al, 2002). Therefore, it appears that tolerance develops to many of the peripheral and central effects of the drug during the period of drug escalation (Fischman and Schuster, 1974; Schmidt et al, 1985a; Cook et al, 1992; Angrist, 1994; Lit et al, 1996). Of particular relevance, this tolerance that occurs during the escalating dose (ED) phase of abuse, likely impacts many other actions of the subsequent high-dose stimulant exposure. Therefore, to more closely approximate highdose METH abuse patterns, including the progressive increase in drug dose that typically precedes high-dose use, we have employed an ED-daily multiple administrations paradigm. Our previous results revealed that this stimulant protocol produced the progressive emergence of a unique behavioral profile and concomitant regionally specific DA response patterns in the striatum (Segal and Kuczenski, 1997a, b, c; Kuczenski and Segal, 1997). These effects appear to be both qualitatively as well as quantitatively different from the responses we have observed with any acute dose or single daily injection pattern of stimulant treatment. Therefore, it is conceivable that pretreatment with an ED regimen might also significantly alter the neurotoxic profile typically associated with subsequent exposure to a single high-dose METH binge. The results of the present study support the hypothesis that, some characteristic behavioral, physiological, and neurochemical indices of METH-induced toxicity are significantly attenuated in this animal model that approximates the ED binge pattern of stimulant abuse.

\section{MATERIALS AND METHODS}

\section{Subjects}

Male Sprague-Dawley rats, weighing $325-350 \mathrm{~g}$ at the beginning of drug treatment, were housed for at least 1 week prior to experimental manipulation in groups of two or three in wire mesh cages, with ad libitum access to food and water, in a temperature- and humidity-controlled room. The room as well as the experimental chambers (see below) were maintained on a reversed 12-h dark (0700-1900), 12-h light cycle to facilitate testing during the normal active phase of the awake/sleeping cycle. During the dark period, all facilities were illuminated with red light to enable accurate observation of the animals. Animals were obtained from Simonsen Labs (Gilroy, CA). All studies adhered to animal welfare guidelines ('Principles of Laboratory Animal Care,' NIH Publication \#85-23).

\section{Apparatus}

Behavior was monitored in custom-designed activity chambers (Segal and Kuczenski, 1987). Briefly, each of the chambers was located in a sound-attenuated cabinet maintained on a reversed 12-h dark 12-h light cycle with constant temperature $\left(24^{\circ} \mathrm{C}\right.$, see below) and humidity $(55 \pm 5 \%)$. Each chamber consisted of two compartments: an activity/exploratory compartment $(30 \times 20 \times 38 \mathrm{~cm})$ and a smaller 'home' compartment $(14 \times 14 \times 10 \mathrm{~cm})$ in which food and water were available ad libitum. Movements of the 
animal between quadrants within the activity/exploratory compartment (ie crossovers) and rearings against the wall, as well as eating and drinking and other vertical (eg contact with a hanging stimulus) and horizontal movements (eg intercompartment crossings) were monitored continuously by computer. In our previous ED-binge treatment studies, observations of animals during the locomotor phase suggested that enhanced locomotion was typically displayed in the form of burst-like patterns in which most crossovers between quadrants were made in rapid succession of one another (ie between 0 and $2 \mathrm{~s}$ ). In order to obtain a quantifiable index of this increased rate of movement, we added to our data collection system the capability of separately monitoring the crossovers made within a $0-2 \mathrm{~s}$ intercrossover interval (ICI), that is, $0-2 \mathrm{~s}$ ICI. These rapid crossovers were separately monitored and presented as a percent of total crossovers (locomotor rate index).

In addition to the computer-monitored behaviors, at least one-half of the animals (selected randomly) were simultaneously videotaped for $60 \mathrm{~s}$ at successive 5 -min intervals for up to $8 \mathrm{~h}$, in order to assess the qualitative features of the response during both the stereotypy and poststereotypy phases. Raters who were unaware of the specific experimental conditions subsequently rated the videotapes on the basis of behavior ethograms and rating procedures established previously (Segal and Kuczenski, 1987). Stereotypy was assessed as the percentage of the observation interval during which the animal displayed each specific behavior. The appearance of other atypical responses or behavior patterns, undetectable by our automated methods, was noted by the rater after each sampling interval.

\section{Drugs}

D-METH hydrochloride (Sigma Chemical Co., St Louis, MO) was dissolved in saline and administered s.c. $(2 \mathrm{ml} / \mathrm{kg}$, to avoid local irritation that might be produced by high concentrations). Doses represent the free base.

\section{General Procedures}

At 3 days prior to the beginning of drug treatment, animals $(n ' s=10)$ were placed in individual experimental chambers, where they remained for the duration of the experiment. To facilitate habituation to the procedures, animals were handled and injected with saline at least once a day. During the remainder of the day and night, animals were not disturbed. Throughout the remaining phases of each study, groups of control animals were administered single daily injections of saline. (In a parallel experiment, separate groups of animals (saline, acute binge, $n$ 's $=7$ ) were maintained under identical treatment conditions, but automated behavioral data were not collected.)

Following the habituation period, groups of animals were initially exposed to the ED phase of drug administration. During this phase, animals received three injections per day of saline or gradually increasing doses of METH for 14 days as summarized in Table 1.

On the day following the ED pretreatment, animals were exposed to the binge treatment consisting of four successive injections of $6.0 \mathrm{mg} / \mathrm{kg} \mathrm{METH}$ at 2-h intervals, beginning at 0900. Preliminary studies indicated that core temperature
Table I ED Pretreatment Schedule

\begin{tabular}{lccc}
\hline & \multicolumn{3}{c}{ METH dose (mg/kg) } \\
\cline { 2 - 4 } & $\mathbf{0 9 0 0}$ & $\mathbf{1 2 1 5}$ & $\mathbf{1 6 3 0}$ \\
\hline Day I & 0.1 & 0.2 & 0.3 \\
Day 2 & 0.4 & 0.5 & 0.6 \\
Day 3 & 0.7 & 0.8 & 0.9 \\
Day 4 & 1.0 & 1.1 & 1.2 \\
Day 5 & 1.3 & 1.4 & 1.5 \\
Day 6 & 1.6 & 1.7 & 1.8 \\
Day 7 & 1.9 & 2.0 & 2.1 \\
Day 8 & 2.2 & 2.3 & 2.4 \\
Day 9 & 2.5 & 2.6 & 2.7 \\
Day 10 & 2.8 & 2.9 & 3.0 \\
Day I1 & 3.1 & 3.2 & 3.3 \\
Day 12 & 3.4 & 3.5 & 3.6 \\
Day 13 & 3.7 & 3.8 & 3.9 \\
Day 14 & 4.0 & 4.0 & 4.0 \\
\hline
\end{tabular}

was significantly elevated at $120 \mathrm{~min}$ after the third METH injection, and assessment of temperature at this time point enabled full characterization of the temporal pattern of the behavioral response to the final METH administration without disturbing the animals. Therefore, core temperature was assessed, and observational behavioral ratings were carried out immediately prior to the fourth injection of the METH binge. At this time, each animal was removed from the behavioral chamber and the presence or absence of salivation, nasal discharge, and/or dark secretions around the eyes was noted. The animal was then placed with its head positioned in the center of a $16^{\prime}$ diameter circle in an open field $\left(24^{\prime} \times 24^{\prime}\right)$, and the number of seconds (up to $30 \mathrm{~s}$ ) for the animal's forepaws to cross the target circle was recorded. The core temperature was then taken with a $2^{\prime}$ flexible probe (YSI Instruments, Model 4600), allowed to equilibrate for $60 \mathrm{~s}$ after insertion. The animal then received the fourth injection of the METH binge or saline and was returned to the behavioral chamber for characterization of the full temporal profile of the behavioral response to the injection.

\section{Ambient Temperature}

The ambient temperature was maintained at $24^{\circ} \mathrm{C}$, comparable to most previous studies of METH-induced toxicity. Consistent with other reports in the literature, our pilot data revealed that a small increase in ambient temperature to $25.5^{\circ} \mathrm{C}$, resulted in a marked decrease in the ability of animals to survive an acute METH $(6 \mathrm{mg} / \mathrm{kg})$ binge: five of seven were dead or prone within $15 \mathrm{~min}$ after the fourth METH injection. We did find, however, that all EDpretreated animals survived the METH binge, and only one exhibited evidence of a toxic reaction, that is, prone at $30 \mathrm{~min}$ after the fourth injection.

\section{Biochemical Analyses}

Animals were killed for tissue analyses of DA and metabolites, and of $\left[{ }^{3} \mathrm{H}\right]$ WIN 35,428 and $\left[{ }^{3} \mathrm{H}\right]$ DTBZ binding 3 days after the binge. For tissue levels of DA and 
metabolites, samples were prepared according to Schmidt et al (1990) and were analyzed using HPLC with electrochemical detection as previously described (Kuczenski et al, 1995).

WIN 35,428 binding assay. Caudate-putamen tissue was sonicated (Vibra-Cell homogenizer) in 100 volumes (w/v) of ice-cold binding buffer $(20 \mathrm{mM}$ sodium phosphate, $0.32 \mathrm{M}$ sucrose, $\mathrm{pH} 7.4$ ), and centrifuged at $40000 \mathrm{~g}$ for $20 \mathrm{~min}$. The resultant pellets were resuspended/recentrifuged and then diluted in $50 \mathrm{w} / \mathrm{v}$ (original wet weight) of ice-cold binding buffer. Aliquots of each membrane suspension (100 $\mu$; approx $80 \mu \mathrm{g}$ protein as determined by the Lowry method) were incubated in triplicate with $\left[{ }^{3} \mathrm{H}\right.$ ]WIN 35,428 (final concentration $5 \mathrm{nM}$ ) for $90 \mathrm{~min}$ at $4^{\circ} \mathrm{C}$; nonspecific binding was determined in the presence of $30 \mu \mathrm{M}$ cocaine. Binding was terminated by rapid filtration through $\mathrm{GF} / \mathrm{B}$ glass fiber filters (presoaked in $20 \mathrm{mM}$ sodium phosphate buffer, $\mathrm{pH}$ 7.4, containing $0.1 \%$ polyethylenimine) that were mounted on a Brandel vacuum cell harvester. The tissues were washed three times with $5 \mathrm{ml}$ of ice-cold sodium phosphate buffer (vacuum flowing), and the filters were placed in $10 \mathrm{ml}$ Ecoscint for liquid scintillation counting.

$\left[{ }^{3} H\right] D T B Z$ binding assay. Caudate-putamen tissue was processed as above except for the following: incubation $(2 \mathrm{~h}$ at room temperature) with $2 \mathrm{nM}\left[{ }^{3} \mathrm{H}\right] \mathrm{DTBZ}$; nonspecific binding determined in the presence of $20 \mu \mathrm{M}$ tetrabenazine.

\section{Data Analysis}

Behavioral and neurochemical data were statistically analyzed using repeated measures ANOVA and $t$-tests with Bonferroni corrections for specific group/time.

\section{RESULTS}

\section{Behavioral/Physiological Measures}

At 2-3 min prior to the fourth injection on the binge day, the appearance and responsiveness of the animals were noted and the core temperature was recorded (see Materials and methods for details). When the chamber door was opened, nonpretreated, METH binge rats $(n=10)$ were engaged in relatively intense focused oral stereotypies (ie biting, gnawing, and/or licking) that continued even while they were being handled. This behavior resumed almost immediately when the animals were individually placed in the center of an open field chamber, from which all but two did not move for the duration of the 30-s test period. In addition, eight of the 10 rats in this group exhibited signs of both excess salivation, evidenced by marked wetness on their chin and neck regions, as well as darkening around the eyes due to excess porphyrin discharge from the Harderian gland.

In contrast, the animals pretreated with the ED protocol prior to the binge exposure $(n=10)$, were markedly different in both appearance and responsivity. None of these rats was engaged in intense stereotypies when the chamber doors were opened, and the milder forms of stereotypy they displayed ceased when these rats were handled. Furthermore, all but one animal moved from the
CORE TEMPERATURE DURING METH BINGE (6.0 mg/kg/2 hrs X 3 INJ.)

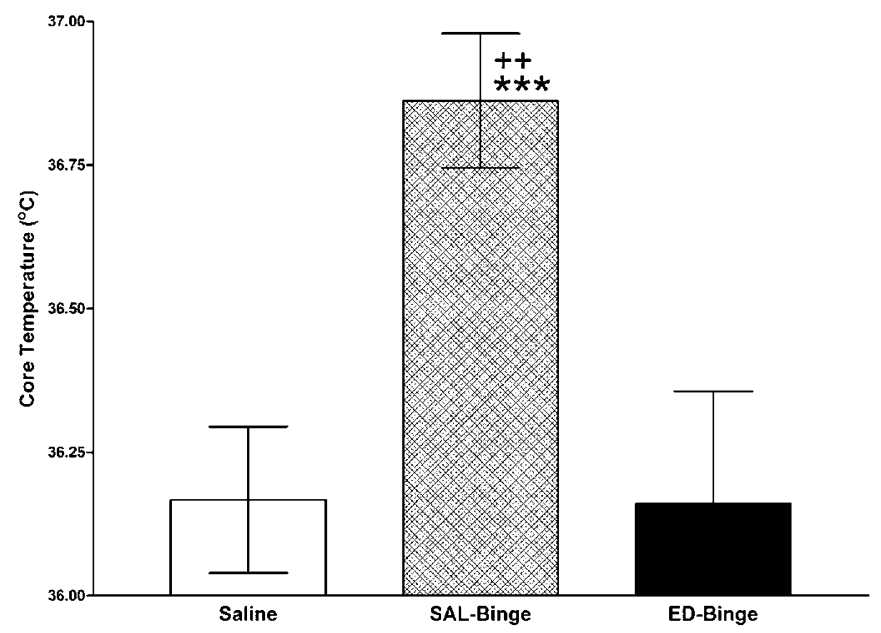

Figure I Effects of ED pretreatment on core temperatures during a $\mathrm{METH}$ binge. Animals were treated with saline (SAL) or increasing doses of METH as described in Materials and methods, then exposed to an METH binge $(6 \mathrm{mg} / \mathrm{kg} / 2 \mathrm{~h} \times 4$ injections). Core temperature values were obtained immediately prior to the fourth injection, and are presented as Mean \pm SEM. **** $P<0.00$ I compared to Saline. ${ }^{++} P<0.0$ I compared to ED binge.

center of the open field within the 30-s trial period (time to exit center: pretreated $v s$ nonpretreated, $12.0 \pm 2.8$ and $28.5 \pm 1.4 \mathrm{~s}, t=5.2 ; \quad P<0.0001)$. Differences between the two groups were also revealed in measures of the core body temperature, which were significantly elevated only in the nonpretreated rats (Figure 1).

The behavioral response to the binge treatment, including both its locomotor (Figure 2) and stereotypy (Figure 3) components, was also profoundly altered by the ED treatment. This difference between ED and saline pretreated groups is most apparent in their comparison after the fourth injection, which allows for a full characterization of the temporal pattern of the behavior. The response of the saline-pretreated rats to the fourth injection of the METH binge consisted primarily of focused oral stereotypies, which appeared within several mins after injection, and included continuous biting, gnawing, and/or licking, primarily directed at the floor of the chamber. This intense form of stereotypy persisted for at least $3 \mathrm{~h}$ in most rats, at which time it was replaced by focused sniffing and/or repetitive head movements for about $1.5 \mathrm{~h}$. Relatively low levels of locomotor activity, occasionally interrupted by progressively more intermittent episodes of mild stereotypy, were exhibited for the remaining $2 \mathrm{~h}$ of the response.

In marked contrast, the focused stereotypy phase of the ED pretreated animals was substantially shorter, that is, about $2-3 \mathrm{~h}$ in duration for most rats in this group and the response contained a significantly greater proportion of the milder forms of the behavior, that is, sniffing and repetitive head movements (Figure 3). In addition, unlike the salinepretreated rats, these animals exhibited a pronounced period of locomotor activation that emerged after the stereotypy phase, and during which there were relatively frequent episodes of 'bursting' locomotion (Figure 2; see Materials and methods for details). 


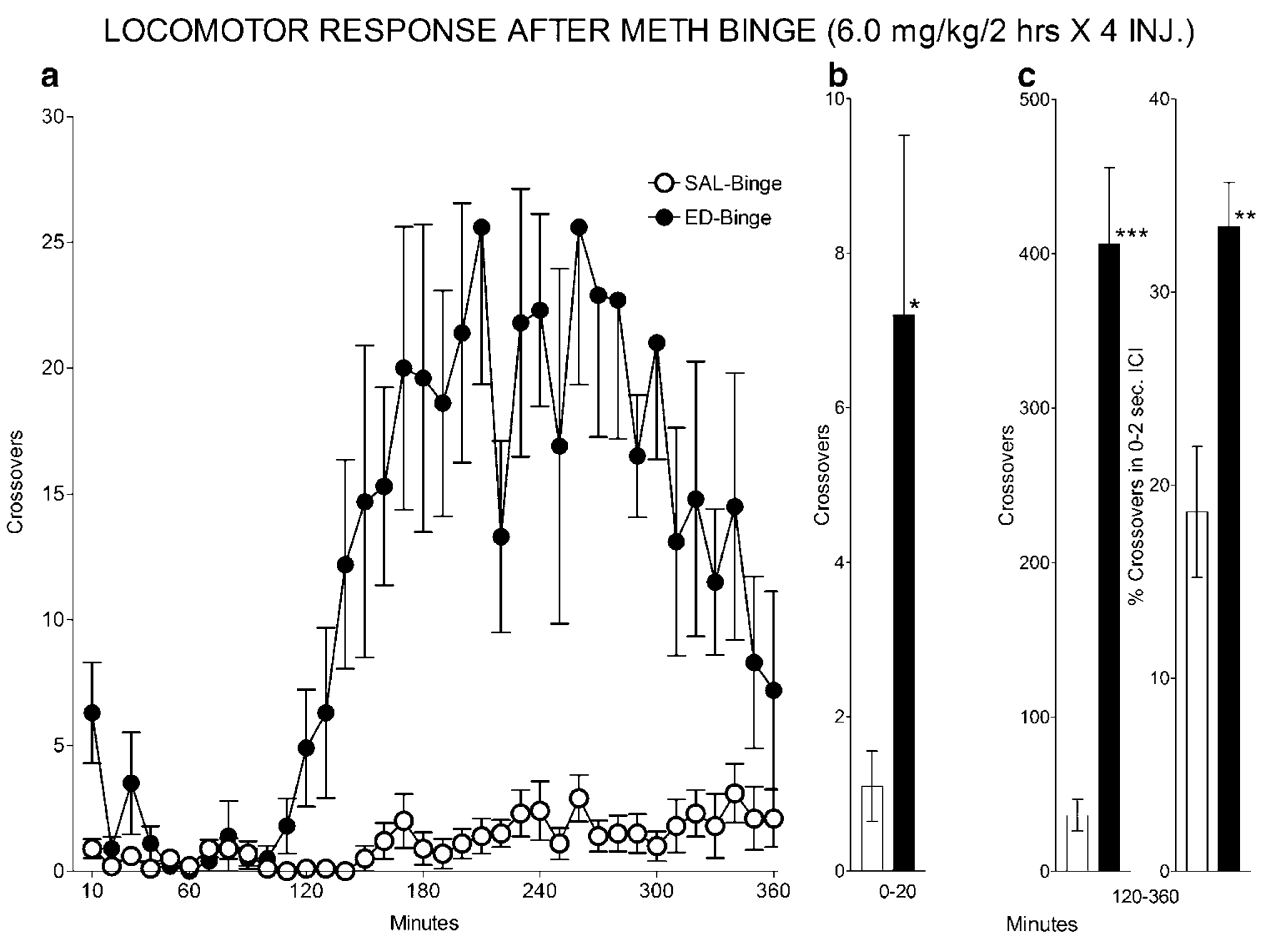

Figure 2 Effects of ED pretreatment on locomotor response monitored after the fourth injection of a METH binge. Animals were treated with saline or increasing doses of METH as described in Materials and methods, then exposed to an METH binge $(6 \mathrm{mg} / \mathrm{kg} / 2 \mathrm{~h} \times 4$ injections). (a) Temporal pattern of locomotion for $6 \mathrm{~h}$ after the fourth injection of the binge. Bar graphs depict the cumulated response over the indicated interval for: (b) prestereotypy crossovers; (c) left, poststereotypy crossovers; and (c) right, percentage of total crossovers occurring within the $0-2 \mathrm{~s} \mathrm{ICl} \mathrm{(see} \mathrm{Materials} \mathrm{and} \mathrm{methods} \mathrm{for}$ details). $* P<0.05$; $* * P<0.0$ I; **** $<0.00$ I compared to the SAL-pretreated group.

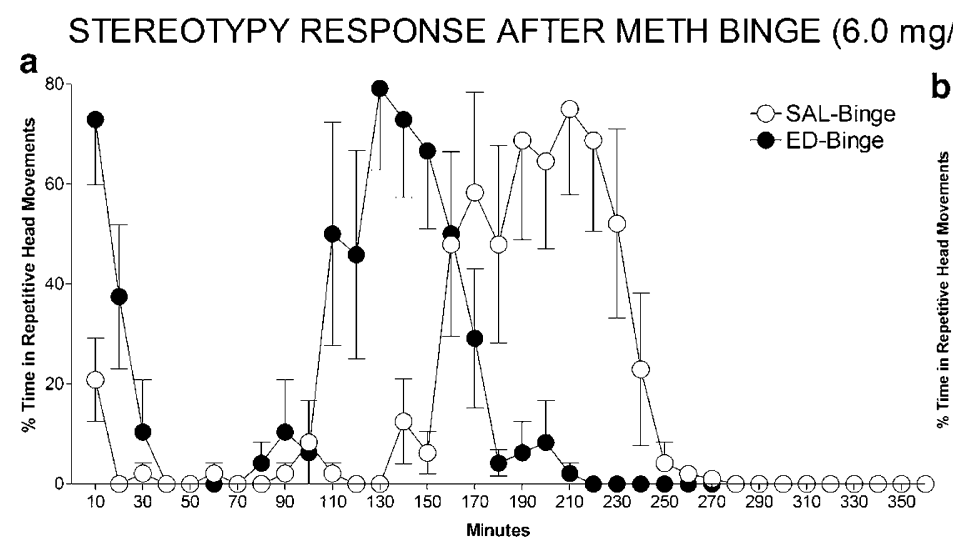

g/kg/2 hrs X 4 INJ.)

a

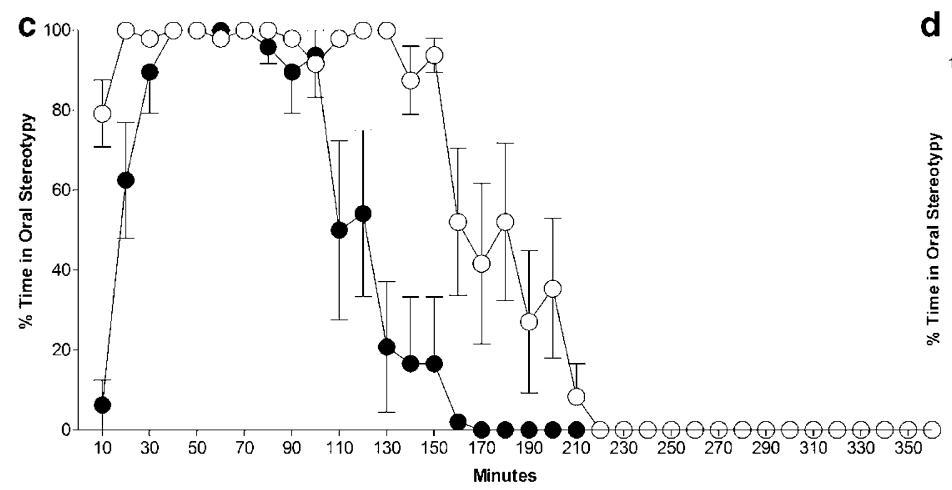

d

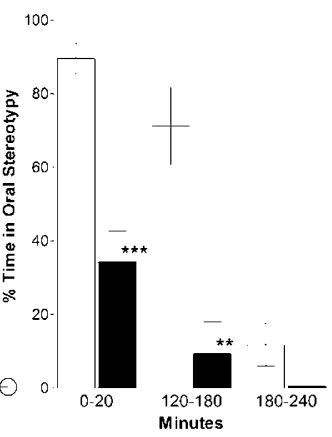

Figure 3 Effects of ED pretreatment on the stereotypy response after the fourth injection of a METH binge. Animals were treated with saline or increasing doses of METH as described in Materials and methods, then exposed to a METH binge $(6 \mathrm{mg} / \mathrm{kg} / 2 \mathrm{~h} \times 4$ injections). (a) Temporal pattern of repetitive head movements. (c) Temporal pattern of oral stereotypy. Bar graphs depict the cumulated response over the indicated interval for: (b) repetitive head movements and (d) oral stereotypy. ${ }^{*} * P<0.01$; ${ }^{*} * * P<0.001$ compared to the SAL-pretreated group. 
Table 2 Effects of ED Pretreatment on METH Binge-Induced Changes in Caudate-Putamen DA Nerve Terminal Markers

\begin{tabular}{|c|c|c|c|c|c|}
\hline & $\begin{array}{l}\text { DA (nmol/g } \\
\text { tissue) }\end{array}$ & $\begin{array}{l}\text { DOPAC (nmol/g } \\
\text { tissue) }\end{array}$ & $\begin{array}{c}\text { HVA (nmol/g } \\
\text { tissue) }\end{array}$ & 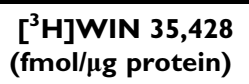 & $\begin{array}{c}\left.{ }^{3} \mathrm{H}\right] \mathrm{DTBZ}(\mathrm{fmol} / \\
\mu \mathrm{g} \text { protein) }\end{array}$ \\
\hline $\begin{array}{l}\text { Control } \\
\text { Acute binge } \\
\text { ED binge }\end{array}$ & $\begin{array}{l}87.7 \pm 2.0 \\
55.1 \pm 4.3 * * * * \\
78.9 \pm 1.7 * \text { ****\#\#\# }\end{array}$ & $\begin{array}{l}10.7 \pm 0.3 \\
8.2 \pm 0.6 \text { **** } \\
11.6 \pm 0.5^{\# \# \#}\end{array}$ & $\begin{array}{l}3.7 \pm 0.1 \\
2.6 \pm 0.2 * * * * \\
3.6 \pm 0.2^{\# \# \#}\end{array}$ & $\begin{array}{l}0.272 \pm 0.033 \\
0.186 \pm 0.019 * * * \\
0.276 \pm 0.029^{\# \#}\end{array}$ & $\begin{array}{l}0.563 \pm 0.02 \\
0.482 \pm 0.02 * * \\
0.489 \pm 0.02 * *\end{array}$ \\
\hline
\end{tabular}

ANOVA; DA: $\mathrm{F}_{[2,41]}=30.5, P<0.00$ I; DOPAC: $\left.\mathrm{F}_{[2,41]}=13.9, \mathrm{P}<0.00 \mathrm{I} ; \mathrm{HVA}: \mathrm{F}_{[2,41]}=20.4, \mathrm{P}<0.00 \mathrm{I} ;{ }_{[3}^{3} \mathrm{H}\right] \mathrm{WIN} 35,428: \mathrm{F}_{[2,41]}=8.46, P<0.00 \mathrm{I} ;\left[{ }^{3} \mathrm{H}\right] \mathrm{DTBZ}$ :

$F_{[2,40]}=6.90, P<0.01$. Control $n=17$; acute binge $n=17$ ( 16 for $\left[{ }^{3} H\right]$ DTBZ); ED binge $n=10$.

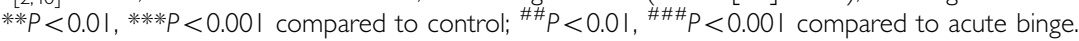

\section{Neurochemical Measures}

To examine the effects of ED pretreatment on the consequences of an METH binge exposure on DA nerve terminal integrity, levels of the transmitter, its metabolites, and the binding of $\left[{ }^{3} \mathrm{H}\right] \mathrm{WIN} 35,428$ to the DAT and $\left[{ }^{3} \mathrm{H}\right] \mathrm{DTBZ}$ to the vesicular transporter were assessed in caudate-putamen 3 days following the final METH injection. The results are summarized in Table 2. The acute METH binge resulted in significant deficits in DA, its metabolites, and levels of $\left[{ }^{3} \mathrm{H}\right]$ WIN 35,428 and $\left[{ }^{3} \mathrm{H}\right] \mathrm{DTBZ}$ binding to caudate-putamen membranes. ED pretreatment significantly attenuated the effects of the METH binge on all parameters with the exception of $\left[{ }^{3} \mathrm{H}\right] \mathrm{DTBZ}$ binding to the vesicular transporter.

\section{DISCUSSION}

Considerable evidence suggests that abuse of the amphetamines in humans often begins with a period of relatively gradual dose escalation, during which tolerance develops to many of the toxic or lethal effects of the high cumulative doses ultimately attained during 'binges' (Zalis and Parmley, 1963; Fischman and Schuster, 1974; Kalant and Kalant, 1975; Schmidt et al, 1985a; Cook et al, 1992; Angrist, 1994; Lit et al, 1996). Since the pattern of drug administration, as well as dose, can significantly influence both the quantitative and qualitative effects of the drug (Gawin and Khalsa, 1996; Unterwald et al, 2001; Riddle et al, 2002a), we suggested that animal model paradigms designed to study the effects of high-dose stimulant abuse should attempt to simulate this pattern of ED exposure (Segal and Kuczenski, 1997a, b, c; Kuczenski and Segal, 1997). In the present study, we demonstrated that ED pretreatment profoundly altered the response to a single binge with high-dose METH, both with respect to striatal DA markers most often assessed in human METH abusers, as well as a behavioral response profile, that is, stereotypy, known to be significantly mediated by METH actions on striatal DA systems. These response changes in ED-pretreated animals were accompanied by the development of tolerance to the hyperthermia and to several other indices of toxicity as evidenced by the pronounced sympathetic activation observed in rats who did not receive this pretreatment prior to the METH binge.

The acute METH binge protocol used in the present study produced significant decreases in a number of striatal DA terminal markers, including DA content (37\% decrease), and DAT and VMAT2 binding (32\% decrease and 15\% decrease, respectively). These effects, which were apparent 3 days following the acute METH binge, are generally comparable to the degree of change reported by others using similar dose regimens administered to rats $(4-6 \mathrm{mg} /$ $\mathrm{kg} / 2 \mathrm{~h} \times 4$ injections). For example, Eisch et al (1996) reported a near $40 \%$ decrease in DAT binding 6-7 days after an acute binge, and Frey et al (1997) found similar reductions in DAT binding as well as a $26 \%$ decrease in VMAT2 binding. In all, $40 \%$ decreases in DA levels are also typical of this dose range (Bowyer and Holson, 1995), although decrements higher than $50 \%$ have also been reported (Cass and Manning, 1999).

Many of the effects associated with the acute bingeinduced neurotoxicity have been shown to persist long after exposure to the drug. However, significant recovery has often been apparent when these markers are assessed at successive withdrawal times. For example, in one recent study (Cass and Manning, 1999), rats treated with METH ( $5 \mathrm{mg} / \mathrm{kg} / 2 \mathrm{~h} \times 4$ injections) were found, 1 week later, to have pronounced changes in a number of striatal DA processes as indicated by decreases in basal DA and its metabolites; evoked overflow of DA and DA clearance, that is, uptake, were also lower. Evoked overflow had partially recovered by 1 month and was at normal levels by 6 months, whereas tissue levels required 12 months to fully recover, at which time all parameters measured had returned to control values. Others have also reported long-term, albeit incomplete, recovery of DA content in the striatum of rats after neurotoxic doses of METH (Bowyer et al, 1992; Friedman et al, 1998). In monkeys, Melega et al (1997) showed that striatal DA levels and DA synthesis capacity, as assessed with PET and $\left[{ }^{18} \mathrm{~F}\right]$ fluoro-LDOPA, exhibited significant recovery at 12 weeks after treatment with METH or AMPH $(2 \mathrm{mg} / \mathrm{kg} / 4 \mathrm{~h} \times 2$ injections). Subsequently, in other PET studies using $\left[{ }^{11} \mathrm{C}\right]$ WIN 35,428 (WIN)-, a cocaine analog that binds to theDAT, they reported that an initial $80 \%$ reduction in WIN binding produced by a similar METH treatment almost fully recovered by 1.5 year (Harvey et al, 2000).

These findings in both rats and infrahuman primates indicate that while the rate of recovery varies widely depending on the specific striatal DA marker, many prominent indices of DA terminal neurotoxicity previously assessed display at least some degree of recovery. In addition, evidence suggests species- and age-differences in the vulnerability to METH and it appears that some indices may be permanently attenuated (ie only partially recover) depending on the extent and severity of the METH treatment (Ojeda et al, 1980; Lucot et al, 1982; Woolverton et al, 1989; Cappon et al, 1997; Cass and Manning, 1999; Kokoshka et al, 2000; Riddle et al, 2002a). However, when recovery has been demonstrated, the differential rates 
apparent for the various markers could indicate that different underlying mechanisms are involved. Thus, the relatively short-term reversal of some of the dopaminergic processes diminished by METH treatment may reflect recovery after initial, transient inhibition or downregulation in response to excessive release of DA (Wilson et al, 1996; Guilarte, 2001). However, Cass and Manning (1999) suggested that the prolonged time period required for full recovery to occur is more consistent with changes initiated by METH-induced degeneration of DA terminals. According to this notion, the relatively rapid recovery of markers is mediated by upregulation in surviving terminals, with the more gradual reversal of METH-induced effects resulting from axonal sprouting and reinnervation of the striatum. Although some indirect evidence supports this notion (Axt et al, 1994), further, more systematic morphometric analyses will be required to accurately determine the possible role of these mechanisms in the recovery process after neurotoxic METH treatment.

In addition to this apparent recovery, which for some measures requires relatively long time periods after exposure to an acute binge, we found that some of the METH-induced effects measured three days after exposure to the binge, were significantly reduced by ED pretreatment: the binge-induced decrease in DA levels was attenuated and the reduction in DAT binding was completely blocked. These findings are consistent with other studies showing that various acute METH binge effects on DA pathways are diminished by prior METH treatment. In previous reports, two different paradigms were used to examine the effects of pretreatment on the neurotoxicity produced by an acute binge. In one series of studies, a sequence of binges with ED $(2-7.5 \mathrm{mg} / \mathrm{kg})$, separated by 1 -day withdrawal periods, served as the METH pretreatment for subsequent challenge with an extremely high-dose METH binge $(15 \mathrm{mg} / \mathrm{kg} / 6 \mathrm{~h} \times 5$ injections) (Schmidt et al, 1985b). These researchers found that the toxic binge-induced reductions in a number of striatal DA and 5HT markers, including the concentration of each of the transmitters as well as their metabolites and rate-limiting enzymes, were either attenuated or prevented in pretreated animals. A similar protective effect was identified for sulpiride binding in both caudate-putamen and nucleus accumbens (Schmidt et al, 1985a). Furthermore, since forebrain levels of METH and AMPH were found to be reduced in METH-pretreated animals, these investigators concluded that dispositional factors were responsible for the tolerance development. Based on the results of more recent follow-up studies designed to examine further the role of pharmacokinetic changes associated with the sequential escalating binge paradigm, this research group concluded that the tolerance is due to changes in the plasma to brain distribution of METH, rather than to enhanced metabolism of the drug (Alburges et al, 1990; Gygi et al, 1996).

In addition to these multi-binge, high-dose studies, the relationship between pretreatment with various sensitization regimens and an acute neurotoxic binge has also been characterized. Stephans and Yamamoto (1996) examined indices of striatal neurotoxicity following a drug $\mathrm{METH}$ protocol of seven single daily injections of METH $(2 \mathrm{mg} / \mathrm{kg})$ and a subsequent exposure to an METH binge challenge $(7.5 \mathrm{mg} / \mathrm{kg} / 2 \mathrm{~h} \times 3$ injections) 1 week after the last pretreat- ment. When measured 4 days following the binge, striatal DA and 5-HT were significantly less depleted in pretreated animals. Using a similar paradigm, Abekawa et al (1997) found that decreases in striatal 5HT, 5HIAA, and DA metabolite levels produced by an METH binge $(5 \mathrm{mg} / \mathrm{kg} /$ $2 \mathrm{~h} \times 4$ injections) were attenuated by AMPH pretreatment ( $4 \mathrm{mg} / \mathrm{kg}$, for 10 days); however, no significant protection was evident in the METH binge-induced reduction of striatal DA content. Finally, Riddle et al (2002a) recently examined the effects of high-dose METH exposure during adolescence ( 6 biweekly, $15 \mathrm{mg} / \mathrm{kg}$ METH injections beginning at postnatal day 40) on several DA markers of neurotoxicity produced by an acute METH binge $(10 \mathrm{mg} /$ $\mathrm{kg} / 2 \mathrm{~h} \times 4$ injections) administered in 90-day old rats. Pretreatment was found to prevent the pronounced bingeinduced decreases in striatal synaptosomal DA uptake and DAT ligand binding. Pharmacokinetic tolerance was ruled out in this study since brain METH concentrations did not differ between pretreated and nonpretreated animals. This observation is consistent with our previous finding of no decrease in brain levels of METH and AMPH as a function of ED pretreatment (Segal and Kuczenski, 1997c). Furthermore, although ED pretreatment does lead to a decrease in the caudate-putamen extracellular DA response and a corresponding attenuated stereotypy response to a subsequent binge (see below for further discussion), the decreased DA response is selective to caudate-putamen DA; that is, neither the nucleus accumbens DA response, nor the hippocampus NE response was attenuated (Segal and Kuczenski, 1997a; Kuczenski and Segal, 1997). Therefore, it appears that drug dispositional changes may occur only when METH pretreatment and/or binge doses are relatively high. Furthermore, particularly when lower doses have been used, the degree of protection has often appeared to be not only brain region- and transmitter-dependent, but also dependent on the specific measure of neurotransmitter function (Segal and Kuczenski, 1987; Eisch et al, 1992; Eisch and Marshall, 1998; Friedman et al, 1998; Wilson et al, 1994; Chapman et al, 2001). Thus, for example, when tested subsequent to the binge, whereas both basal DA and the stimulant-induced extracellular DA response are attenuated by an acute binge (Cass and Manning, 1999), only basal DA is protected when the binge is preceded by ED pretreatment (Segal and Kuczenski, 1997a,b,c; Kuczenski and Segal, 1997). These observations support the view that multiple mechanisms are implicated in the spectrum of effects observed in these studies.

One possible mechanism for the tolerance exhibited by at least some components of the neurotoxic profile may relate to the pronounced sympathetic activation produced by METH. In this regard, a number of studies have examined the possible role of METH-induced hyperthermia in the neurotoxicity produced by exposure to a single, high-dose METH binge regimen. Although some evidence does not support a causal relationship, it appears that under many conditions, hyperthermia, perhaps in combination with other factors, is significantly implicated in the DA neurotoxicity produced by METH (Bowyer et al, 1994; Albers and Sonsalla, 1995; Ali et al, 1996; Cappon et al, 1997; Kuperman et al, 1997; Callahan and Ricaurte, 1998; Malberg and Seiden, 1998; Sandoval et al, 2000; Yuan et al, 2001; Riddle et al, 2002a). Tolerance to effects such as 
hyperthermia would then be expected to result in a corresponding attenuation of the susceptibility to METHinduced neurotoxicity. Our measures of DA content and DAT binding are consistent with such a relationship and, in fact, a similar interpretation was recently proposed by Riddle et al (2002a), based on their study of the pretreatment effects of chronic METH exposure initiated in adolescent rats. It should be noted, however, that particularly with respect to the ED regimen used in our study, the changes in DA markers and hyperthermia observed in pretreated animals may simply reflect parallel, but independent effects of ED pretreatment on different central and peripheral responses to the binge regimen. Consistent with this interpretation, we previously found that the caudate-putamen extracellular DA response to an acute binge is significantly reduced by ED pretreatment (Segal and Kuczenski, 1997a; Kuczenski and Segal, 1997). If the acute binge-induced decreases in DA and DAT binding levels reflect, at least in part, compensatory adjustments initiated by excessive release of caudate DA, then the diminished DA release after ED pretreatment would be expected to produce a correspondingly reduced activation of these compensatory processes. Alternatively, a decrease in DA available for METH-induced release in ED-pretreated animals could be responsible for the attenuated neurotoxic effects by producing less terminal damage. In fact, some evidence suggests that METH-induced excesses in DA, perhaps converted into reactive oxygen species either intraor extra-neuronally, may be related to degeneration of DA terminals (LaVoie and Hastings, 1999; Fleckenstein et al, 2000; Davidson et al, 2001; Larsen et al, 2002). It should be noted, however, that METH-induced hyperthermia also occurs in the form of increased regional brain temperatures (Clausing and Bowyer, 1999). Such local temperature changes may be of sufficient magnitude to alter a number of neurochemical processes, including those that modulate DA release. Therefore, tolerance to these hyperthermic effects could be responsible for the attenuated METHinduced DA release after ED pretreatment. Further studies are required to identify the precise role of temperature changes in METH-induced DA release.

Regardless of the mechanisms underlying tolerance development, our results show that ED pretreatment markedly diminishes the effect of an acute METH binge on DA and DAT levels. Since both of these measures are commonly used markers for DA neurotoxicity, it could be concluded that under conditions which approximate a common pattern of METH abuse in humans, DA terminal degeneration may be marginal, if it occurs at all. In fact, on the basis of their post-mortem study of additional DA terminal markers in chronic METH abusers, Wilson et al (1996) suggested that METH did not result in DA terminal damage.

However, our finding that the acute METH binge-induced decrement in VMAT2 binding was not reversed by ED pretreatment is not consistent with this interpretation, since it has been argued that changes in the vesicular transporter may more accurately reflect the integrity of nigrostriatal DA terminals (Naudon et al, 1994; Vander Borght et al, 1995; Wilson and Kish, 1996; Wilson et al, 1996; Frey et al, 1997; Guilarte, 2001). In contrast, alterations in other measures such as DA and DAT levels may occur as a consequence of either compensatory adjustment or terminal damage (Wiener et al, 1989; Kilbourn et al, 1992; Wilson et al, 1996; Frey et al, 1997; Guilarte, 2001; Little et al, 2003). In addition, as noted above, upregulation in surviving neurons, along with subsequent neuronal sprouting may be responsible for the different times required for DA markers to recover after neurotoxic METH exposure (Cass and Manning, 1999). In this regard, the gradual neuronal sprouting could account for the apparent recovery of VMAT2 that has been observed in primates 1.5 years after brief exposure to METH $(2 \times 2 \mathrm{mg} / \mathrm{kg}, 6 \mathrm{~h}$ apart) (Harvey et al, 2000). It is important to note, however, that while the VMAT2 marker has been considered by many to be an index of DA terminal integrity, it is also possible that some METH effects on this transporter reflect selective damage to the vesicle in the absence of terminal degeneration, as is evident following reserpine administration, which would then require synthesis and axonal transport of new vesicles for eventual full recovery of vesicular-dependent functions (Häggendal and Lindqvist, 1964). Furthermore, recent evidence suggests relatively rapid METH-induced changes in the subcellular distribution of VMAT2 (as well as DAT) (Riddle et al, 2002b; Sandoval et al, 2003). Although these changes appear to be reversed within $24 \mathrm{~h}$ of drug administration, their potential contribution to more persistent METH-induced changes remain to be determined.

Regardless of the mechanisms involved, our data confirm and extend the important observation that tolerance to at least some of the neurotoxic effects produced by moderate to high-dose binge protocols, is conferred by a relatively wide range of METH pretreatment regimens. It is also apparent from other chronic stimulant studies that the specific qualitative nature, persistence, and degree of the neurochemical and behavioral change produced by METH administration likely depend not only on the characteristics of the binge itself, but also on the pattern and conditions associated with the pretreatment regimen (Schmidt et al, 1985b; Unterwald et al, 2001; Riddle et al, 2002a). Therefore, we have argued that animal models designed to examine the consequences of stimulant abuse should incorporate a relatively long period of gradually EDs prior to binge exposure. In our study, we have attempted to approximate this pattern to identify more accurately the neurochemical and behavioral changes relevant to those that occur as a consequence of high-dose METH abuse.

\section{ACKNOWLEDGEMENTS}

This research was supported by NIH Grants DA-01568, DA02854 and a fellowship (DA 14449) to MLO.

\section{REFERENCES}

Abekawa T, Ohmori T, Koyama T (1997). Tolerance to the neurotoxic effect of methamphetamine in rats behaviorally sensitized to methamphetamine or amphetamine. Brain Res 767: 34-44.

Albers DS, Sonsalla PK (1995). Methamphetamine-induced hyperthermia and dopaminergic neurotoxicity in mice: pharmacological profile of protective and nonprotective agents. $J$ Pharmacol Exp Ther 275: 1104-1114. 
Alburges ME, Hanson GR, Gibb JW (1990). Role of methamphetamine metabolism in the development of CNS tolerance to the drug. Invest Clin 31: 165-176.

Ali SF, Newport GD, Slikker WJ (1996). Methamphetatine-induced dopaminergic toxicity in mice. Role of environmental temperature and pharmacological agents. Ann NY Acad Sci 801: 187-198.

Angrist B (1994). Amphetamine psychosis: clinical variations of the syndrome. In: Cho AK, Segal DS (eds) Amphetamine and its Analogues. Academic Press: San Diego. pp 387-414.

Axt KJ, Mamounas LA, Molliver ME (1994). Structural features amphetamine neurotoxicity in the brain. In: Cho AK, Segal DS (eds) Amphetamine and its Analogs: Psychopharmacology, Toxicology and Abuse. Academic: San Diego. pp 315-367.

Bowyer JF, Davies DL, Schmued L, Broening HW, Newport GD, Slikker W et al (1994). Further studies if the role of hyperthermia in methamphetamine neurotoxicity. J Pharmacol Exp Ther 268: 1571-1580.

Bowyer JF, Holson RR (1995). Methamphetamine and amphetamine neurotoxicity. In: Chang LW, Dyer RS (eds) Handbook of Neurotoxicology. Marcel Dekker, Inc.: New York. pp 845-870.

Bowyer JF, Tank AW, Newport GD, Slikker Jr W, Ali SF, Holson RR (1992). The influence of environmental temperature on the transient effects of methamphetamine on dopamine levels in rat striatum. J Pharmacol Exp Ther 260: 817-824.

Callahan BT, Ricaurte GA (1998). Effect of 7-nitroindazole on body temperature and methamphetamine-induced dopamine toxicity. NeuroReport 9: 2691-2695.

Cappon GD, Morford L, Vorhees CV (1997). Ontogeny of methamphetamine-induced neurotoxicity and associated hyperthermic response. Dev Brain Res 103: 155-162.

Cass WA (1997). Decreases in evoked overflow of dopamine in rat striatum after neurotoxic doses of methamphetamine. J Pharmacol Exp Ther 280: 105-113.

Cass WA, Manning MW (1999). Recovery of presynaptic dopaminergic functioning in rats treated with neurotoxic doses of methamphetamine. J Neurosci 19: 7653-7660.

Chang L, Ernst T, Speck O, Patel H, DeSilva M, Leonido-Yee M et al (2002). Perfusion MRI and computerized cognitive test abnormalities in abstinent methamphetamine users. Psychiatry Res Neuroimaging 114: 65-79.

Chapman DE, Hanson GR, Kesner RP, Keefe KA (2001). Long-term changes in basal ganglia function after a neurotoxic regimen of methamphetamine. J Pharmacol Exp Ther 296: 520-527.

Clausing P, Bowyer JF (1999). Time course of brain temperature and caudate/putamen microdialysate levels of amphetamine and dopamine in rats after multiple doses of D-amphetamine. Ann NY Acad Sci 890: 495-504.

Cook CE, Jeffcoat AR, Sadler BM, Hill JM, Voyksner RD, Pugh DE et al (1992). Pharmacokinetics of oral methamphetamine and effects of repeated daily dosing in humans. Drug Metab Dispos 20: $856-862$.

Davidson C, Gow AJ, Lee TH, Ellinwood EH (2001). Methamphetamine neurotoxicity: necrotic and apoptotic mechanisms and relevance to human abuse and treatment. Brain Res Revs 36: $1-22$.

Eisch AJ, Gaffney M, Weihmuller FB, O’Dell SJ, Marshall JF (1992). Striatal subregions are differentially vulnerable to the neurotoxic effects of methamphetamine. Brain Res 598: 321-326.

Eisch AJ, Marshall JF (1998). Methamphetamine neurotoxicity: dissociation of striatal dopamine terminal damage from parietal cortical cell body injury. Synapse 30: 433-445.

Eisch AJ, O’Dell SJ, Marshall JF (1996). Striatal and cortical NMDA receptors are altered by a neurotoxic regimen of methamphetamine. Synapse 22: 217-225.

Farfel GM, Seiden LS (1994). Role of hypothermia in the mechanism of protection against serotonergic toxicity. II. Experiments with methamphetamine, $p$-chloroamphetamine, fenfluramine, dizocilpine and dextromethorphan. J Pharmacol Exp Ther 272: 868-875.

Fischman MW, Schuster CR (1974). Tolerance development to chronic methamphetamine intoxication in the rhesus monkey. Pharmacol Biochem Behav 2: 503-508.

Fleckenstein AE, Gibb JW, Hanson GR (2000). Differential effects of stimulants on monoaminergic transporters: pharmacological consequences and implications for neurotoxicity. Eur J Pharmacol 406: 1-13.

Frey KA, Kilbourn MR, Robinson TE (1997). Reduced striatal vesicular monoamine transporters after neurotoxic but not after behaviorally-sensitizing doses of methamphetamine. Eur $J$ Pharmacol 334: 273-279.

Friedman SD, Castañeda E, Hodge GK (1998). Long-term monoamine depletion, differential recovery, and subtle behavioral impairment following methamphetamine-induce neurotoxicity. Pharmacol Biochem Behav 61: 35-44.

Gawin FH, Khalsa ME (1996). Sensitization and 'street' stimulant addiction. In: Majewska MD (ed) Neurotoxicity and Neuropathology Associated with Stimulant Abuse. NIDA Research Monograph Series. US Government Printing Office: Washington, DC. pp 224-250.

Guilarte TR (2001). Is methamphetamine abuse a risk factor in parkinsonism? Neurotoxicology 22: 725-731.

Gygi MP, Gygi SP, Johnson M, Wilkins DG, Gibb JW, Hanson GR (1996). Mechanisms for tolerance to methamphetamine effects. Neuropharmacology 35: 751-757.

Häggendal J, Lindqvist M (1964). Brain monoamine levels and behavior during long-term administration of reserpine. Int $J$ Neuropharmacol 3: 59-64.

Harvey DC, Lacan G, Tanious SP, Melega WP (2000). Recovery from methamphetamine induced long-term nigrostriatal dopaminergic deficits without substantia nigra cell loss. Brain Res 871: 259-270.

Hogan KA, Staal RGW, Sonsalla PK (2000). Analysis of VMAT2 binding after methamphetamine or MPTP treatment: disparity between homogenates and vesicle preparations. J Neurochem 74: 2217-2220.

Huber A, Ling W, Shoptaw S, Gulati V, Brethen P, Rawson RA (1997). Integrating treatments for methamphetamine abuse: a psychosocial perspective. J Addict Dis 16: 41-50.

Kalant H, Kalant OJ (1975). Death in amphetamine users: causes and rates. Can Med Assoc J 112: 299-304.

Kilbourn MR, Sherman PS, Pisani T (1992). Repeated reserpine administration reduces in vivo [18F]-GBR-13119 binding to the dopamine uptake site. Eur J Pharmacol 216: 109-112.

Kokoshka JM, Fleckenstein AE, Wilkins DG, Hanson GR (2000). Age-dependent differential responses of monoaminergic systems to high doses of methamphetamine. J Neurochem 75: 2095-2102.

Kramer JC (1972). Introduction to amphetamine abuse. In: Ellinwood Jr EH, Cohen S (eds) Current Concepts on Amphetamine Abuse. US Government Printing Office: Washington, DC. pp 177-184.

Kuczenski R, Segal DS (1997). An escalating dose-high dose binge pattern of amphetamine administration results in differential changes in the extracellular dopamine response profiles in caudate-putamen and nucleus accumbens. J Neurosci 17: 4441-4447.

Kuczenski R, Segal DS, Cho AK, Melega WP (1995). Hippocampus norepinephrine, caudate dopamine and serotonin, and behavioral responses to the stereoisomers of amphetamine and methamphetamine. J Neurosci 15: 1308-1317.

Kuperman DI, Freyaldenhoven TE, Schmued L, Ali SF (1997). Methamphetamine-induced hyperthermia in mice: examination of dopamine depletion and heat-shock protein induction. Brain Res 771: 1-9.

Larsen KE, Fon EA, Hastings TG, Edwards RH, Sulzer D (2002). Methamphetamine-induced degeneration of dopaminergic neu- 
rons involves autophagy and upregulation of dopamine synthesis. J Neurosci 22: 8951-8960.

LaVoie MJ, Hastings TG (1999). Dopamine quinone formation and protein modification associated with the striatal neurotoxicity of methamphetamine: evidence against a role for extracellular dopamine. J Neurosci 19: 1484-1491.

Lit E, Wiviott-Tishler L, Wong S, Hyman SE (1996). Stimulants: amphetamines and caffeine. In: Friedman L, Fleming NF, Roberts DH, Hyman SE (eds) Source Book of Substance Abuse and Addiction. Willams \& Wilkins: Baltimore, MD. pp 231-242.

Little KY, Krolewski DM, Zhang L, Cassin BJ (2003). Loss of striatal vesicular monoamine transporter protein (VMAT2) in human cocaine users. Am J Psychiatry 160: 47-55.

Lucot JB, Wagner GC, Schuster CR, Seiden LS (1982). Decreased sensitivity of rat pups to long-lasting dopamine and serotonin depletions produced by methamphetamine. Brain Res 247: 183.

Malberg JE, Seiden LS (1998). Small changes in ambient temperature cause large changes in 3,4-methylenedioxymethamphetamine (MDMA)-induced serotonin neurotoxicity and core body temperature in the rat. J Neurosci 18: 5086-5094.

McCann UD, Wong DF, Yokoi F, Villemagne V, Dannals RF, Ricaurte GA (1998). Reduced striatal dopamine transporter density in abstinent methamphetamine and methcathinone users: evidence from positron emission tomography studies with $\left[{ }^{11}\right.$ C]WIN-35,428. J Neurosci 18: 8417-8422.

Melega WP, Raleigh MJ, Stout DB, Lacan G, Huang SC, Phelps ME (1997). Recovery of striatal dopamine function after acute amphetamine- and methamphetamine-induced neurotoxicity in the vervet monkey. Brain Res 766: 113-120.

Naudon L, Leroux-Nicollet I, Costentin J (1994). Short-term treatments with haloperidol or bromocriptine do not alter the density of the monoamine vesicular transporter in the substantia nigra. Neurosci Lett 173: 1-4.

O'Callaghan JP, Miller DB (2000). Neurotoxic effects of substituted amphetamine in rats and mice: challenge to the current dogma. In: Massaro E, Broderick PA (eds) Handbook of Neurotoxicity. Humana Press: New York.

Ojeda S, Andrews W, Advis J, White S (1980). Recent advances in the endocrinology of puberty. Endocr Rev 1: 228-257.

$\mathrm{Pu}$ C, Vorhees CV (1995). Protective effects of MK-801 on methamphetamine-induced depletion of dopaminergic and serotonergic terminals and striatal astrocytic response: an immunohistochemical study. Synapse 19: 97-104.

Riddle EL, Kokoshka JM, Wilkins DG, Hanson GR, Fleckenstein AE (2002a). Tolerance to the neurotoxic effects of methamphetamine in young rats. Eur J Pharmacol 435: 181-185.

Riddle EL, Topham MK, Haycock JW, Hanson GR, Fleckenstein AE (2002b). Differential trafficking of the vesicular monoamine transporter-2 by methamphetamine and cocaine. Eur J Pharmacol 449: 71-74.

Sandoval V, Hanson GR, Fleckenstein AE (2000). Methamphetamine decreases mouse striatal dopamine transporter activity: roles of hyperthermia and dopamine. Eur J Pharmacol 409: 265-271.

Sandoval V, Riddle EL, Hanson GR, Fleckenstein AE (2003). Methylphenidate alters vesicular monoamine transport and prevents methamphetamine-induced dopaminergic deficits. $J$ Pharmacol Exp Ther 304: 1181-1187.

Schmidt CJ, Gehlert DR, Peat MA, Sonsalla PK, Hanson GR, Wamsley JK et al (1985a). Studies on the mechanism of tolerance to methamphetamine. Brain Res 343: 305-313.

Schmidt D, Roznoski M, Ebert MH (1990). Qualitative and quantitative high performance liquid chromatographic analysis of monoamine neurotransmitters and metabolites in cerebrospinal fluid and brain tissue using reductive electrochemical detection. Biomed Chromatogr 4: 215-220.

Schmidt CJ, Sonsalla PK, Hanson GR, Peat MA, Gibb JW (1985b). Methamphetamine-induced depression of monoamine synthesis in the rat: development of tolerance. J Neurochem 44: 852-855.

Segal DS, Kuczenski R (1987). Individual differences in responsiveness to single and repeated amphetamine administration: behavioral characteristics and neurochemical correlates. $J$ Pharmacol Exp Ther 242: 917-926.

Segal DS, Kuczenski R (1997a). An escalating dose 'binge' model of amphetamine psychosis: behavioral and neurochemical characteristics. J Neurosci 17: 2551-2566.

Segal DS, Kuczenski R (1997b). Behavioral alterations induced by an escalating dose-binge pattern of cocaine administration. Behav Brain Res 88: 251-260.

Segal DS, Kuczenski R (1997c). Repeated binge exposure to amphetamine and methamphetamine: behavioral and neurochemical characterization. J Pharmacol Exp Ther 282: 561-573.

Segal DS, Kuczenski R (1999a). Escalating dose-binge treatment with methylphenidate: role of serotonin in the emergent behavioral profile. J Pharmacol Exp Ther 291: 19-30.

Segal DS, Kuczenski R (1999b). Escalating dose/binge stimulant exposure: relationship between emergent behavioral profile and differential caudate-putamen/nucleus accumbens dopamine responses. Psychopharmacology 142: 182-192.

Seiden LS, Ricaurte GA (1987). Neurotoxicity of methamphetamine and related drugs. In: Meltzer HY (ed) Psychopharmacology: The Third Generation of Progress. Raven Press: New York. pp 359-366.

Seiden LS, Sabol KE (1995). Neurotoxicity of methamphetaminerelated drugs and cocaine. In: Chang LW, Dyer RS (eds) Handbook of Neurotoxicology. Marcel Dekker, Inc.: New York. pp 825-843.

Sekine Y, Iyo M, Ouchi Y, Matsunaga T, Tsukada H, Okada $\mathrm{H}$ et al (2001). Methamphetamine-related psychiatric symptoms and reduced brain dopamine transporters studied with PET. Am J Psychiatry 158: 1206-1214.

Sekine Y, Minabe Y, Kawai M, Suzuki K, Iyo M, Isoda $\mathrm{H}$ et al (2002). Metabolite alterations in basal ganglia associated with methamphetamine-related psychiatric symptoms: a proton MRS study. Neuropsychopharmacology 27: 453-461.

Simon SL, Rawson RA, Dacey J, Glynn S, Domier CP (2000). A comparison of patterns of methamphetamine and cocaine use. Drug Alcohol Depend.

Simon SL, Richardson K, Dacey J, Glynn S, Domier CP, Rawson RA et al (2002). A comparison of patterns of methamphetamine and cocaine use. J Addict Dis 21: 35-44.

Sonsalla PK, Heikkila RE (1988). Neurotoxic effects of 1-methyl-4phenyl-1,2,3,6-tetrahydropyridine (MPTP) and methamphetamine in several strains of mice. Prog Neuropsychopharmacol Biol Psychiatry 12: 345-354.

Stephans SE, Yamamoto BK (1996). Methamphetamine pretreatment and the vulnerability of the striatum to methamphetamine neurotoxicity. Neuroscience 72: 593-600.

Unterwald EM, Kreek MJ, Cuntapay M (2001). The frequency of cocaine administration impacts cocaine-induced receptor alterations. Brain Res 900: 103-109.

Vander Borght TM, Kilbourn MR, Desmond T, Kuhl D, Frey K (1995). The vesicular monoamine transporter is not regulated by dopaminergic drug treatments. Eur J Pharmacol 294: 577-583.

Villemagne V, Yuan J, Wong DF, Dannals RF, Hatzidimitriou G, Mathews WB et al (1998). Brain dopamine neurotoxicity in baboons treated with doses of methamphetamine comparable to those recreationally abused by humans: evidence from $\left[{ }^{11} \mathrm{C}\right]$ WIN-35,428 positron emission tomography studies and direct in vitro determinations. J Neurosci 18: 419-427.

Volkow ND, Chang LW, Wang GJ, Fowler JS, Franceschi D, Gatley SJ et al (1999). In vivo evidence that methamphetamine abuse produces long lasting changes in dopamine transporters in human brain. J Nuclear Med 40(Suppl): 110P. 
Volkow ND, Chang L, Wang GJ, Fowler JS, Franceschi D, Sedler M] et al (2001a). Higher cortical and lower subcortical metabolism in detoxified methamphetamine abusers. Am J Psychiatry 158: 383-389.

Volkow ND, Chang L, Wang GJ, Fowler JS, Franceschi D, Sedler MJ et al (2001b). Loss of dopamine transporters in methamphetamine abusers recovers with protracted abstinence. J Neurosci 21: 9414-9418.

Wiener LH, Hashim A, Lajtha A, Sershen H (1989). Chronic Ldeprenyl-induced up-regulation of the dopamine uptake carrier. Eur J Pharmacol 163: 194.

Wilson JM, Kalasinsky KS, Levey AI, Bergeron C, Reiber G, Anthony RM et al (1996). Striatal dopamine nerve terminal markers in human, chronic methamphetamine users. Nat Med 2: 699-703.

Wilson JM, Kish SJ (1996). The vesicular monoamine transporter, in contrast to the dopamine transporter, is not altered by chronic cocaine self-administration in the rat. J Neurosci 16: 3507-3510.

Wilson JM, Nobrega JN, Carroll ME, Niznik HB, Shannak K, Lac ST (1994). Heterogeneous subregional binding patterns of $3 \mathrm{H}-\mathrm{WIN}-35$ and $3 \mathrm{H}-\mathrm{GBR} 12,935$ are differentially regulated by chronic cocaine self-administration. J Neurosci 14: 2966-2979.

Woolverton WL, Ricaurte GA, Forno LS, Seiden LS (1989). Longterm effects of chronic methamphetamine administration in rhesus monkeys. Brain Res 486: 73-78.

Wrona MZ, Yang Z, Zhang F, Dryhurst G (1997). Potential new insights into the molecular mechanisms of methamphetamine-induced neurodegeneration. NIDA Res Monogr 173: 146-174.

Xie T, McCann UD, Kim S, Yuan J, Ricaurte GA (2000). Effect of temperature on dopamine transporter function and intracellular accumulation of methamphetamine: implications for methamphetamine-induced dopaminergic neurotoxicity. J Neurosci 20: 7838-7845.

Yuan J, Callahan BT, McCann UD, Ricaurte GA (2001). Evidence against an essential role of endogenous brain dopamine in methamphetamine-induced dopaminergic neurotoxicity. $J \mathrm{Neu}$ rochem 77: 1338-1347.

Zalis EDG, Parmley LF (1963). Fatal amphetamine poisoning. Arch Intern Med 112: 60-64. 